Learning Objectives: Shed some light on role of MRI for cholesteatoma.

Background: Although the diagnosis of cholesteatoma is in nearly all cases achieved by a meticulous otomicroscopical or endoscopical examination, imaging is usually required for a better definition of the extension of the pathology as well as to evidentiate eventual bony erosions. Non ECHOplanar diffusion weighted magnetic resonance (non-EPI DW MRI) has been recently acquired as an important diagnostic tool in case of cholesteatoma, with high rates of sensitivity and specificity. At the ENT Unit of Sant'Andrea Hospital in Rome, Italy, this technique is regularly applied since five years especially for following-up after surgery. In this study, specific protocols are presented to be applied in different clinical situations.

Material and Methods: A consecutive number of subjects affected by cholesteatoma were scheduled for surgery. Both primary and recurrent cases were taken into consideration. Primary cases were subdivided in limited and extended cases, while recurrent cases comprised both routine cases and sequels from subtotal petrosectomy with blind sac closure of the external meatus. In the extended cases and in petrous bone cholesteatoma cases, non-EPI DW MRI was planned soon after surgery (within 1 month) and 3, 6 and 12 months after surgery. In the limited cases, it was only planned 12 months after surgery.

Results and Discussion: Non-EPI DW MRI has proven to be highly sensitive for detecting residual pathology with only rare cases of false positivity. The early application of this technique in selected invasive cases enabled to reassure the surgeon on the performed surgical procedure or give notice of the expected residual tissue left in particular cases where other priorities were taken into consideration (e.g, facial nerve function).

doi:10.1017/S0022215116005077

\section{ID: IP011}

\section{Intracranial complications of chronic otitis media}

Presenting Author: Anna Bartochowska

Anna Bartochowska, Lukasz Borucki, Agata Buczkowska, Witold Szyfter

Poznań University of Medical Sciences

Learning objectives: In the presentation the clinical course and therapeutic results of intracranial complications of otogenic origin will be discussed.

Introduction: Intracranial complications of otogenic origin are rare but still represent a potentially lethal threat.

Methods: The aim of the study was clinical analysis of 48 intracranial complications in 29 patients with chronic otitis media. The incidence of complications, symptoms reported at admission, neurological condition, microbiological material, the choice of the surgical therapeutic method and treatment results were evaluated.
Results: $16 / 29$ patients had a single complication, while 13/ 29 - multiple complications. Brain abscess was reported in $18 / 48$ cases, meningitis in $14 / 48$, sigmoid sinus thrombosis in $7 / 48$, epidural abscess in $6 / 48$ and subdural empyema in $3 / 48$. Surgical treatment was implemented immediately and simultaneously in the temporal bone focus and the site of complication. There were no deaths.

Conclusions: Brain abscess was the most common complication in the study group. In many patients several intracranial complications occurred at the same time. The authors recommend fast evacuation of the temporal bone purulent focus accompanied by the surgical treatment of intracranial complications with the evacuation of the abscess under the control of neuronavigation.

\section{doi:10.1017/S0022215116005089}

\section{ID: IP012}

Endoscopic Ear Surgery and its impact on the operating theatre team

\section{Presenting Author: Paramita Baruah}

\author{
Paramita Baruah ${ }^{1}$, Duncan Bowyer ${ }^{2}$ \\ ${ }^{1}$ West Midlands Deanery, ${ }^{2}$ Princess Royal \\ Hospital Telford
}

\section{Learning Objectives:}

Introduction: The development of Endoscopic Ear Surgery (EES), from being an adjunct to microscopic dissection to becoming the prime methodology in select cases, has been an exciting recent development. This work assesses the experience of theatre team members with EES versus conventional ear surgery.

Methodology: A questionnaire was designed covering the areas of theatre time management (planning and organisation, leadership and direction, inter-team working), team thinking (shared situational understanding, thinking ahead, decision making) and team safety (safe practice, equipment use, low energy and fatigue), comparing EES to conventional microsurgery of the ear. The scale used was: 1-much worse, 2-somewhat worse, 3-neither better nor worse, 4-somewhat better, 5-much better.

Results: The respondents included 7 theatre nurses, 3 anaesthetists and 3 theatre practitioners. All respondents reported a greater subjective satisfaction with EES mainly with regard to being able to appreciate what was happening during the surgery. The anaesthetists reported that it was easier to anticipate anaesthetic requirements at the close of the procedure in EES and that patients were more comfortable postoperatively. Five out of the seven nurses consistently rated EES as a 4 or 5 compared to conventional ear surgery with regard to theatre team management, team thinking and team safety. EES was initially perceived as challenging by the nurses but with experience they report a greater degree of involvement and satisfaction with the endoscopic procedure. The theatre practitioners rated EES to be better in theatre team management and team thinking but as equivocal with regard to team safety. 
Conclusion: Theatre staff report an initial challenging learning curve with EES. With time however the theatre team satisfaction levels are higher due to greater awareness and involvement with the surgical procedure.

Learning points: The theatre team shows high levels of satisfaction with EES.

doi:10.1017/S0022215116005090

\section{ID: IP013}

Sonotubometry using perfect sequences: clinical results of the $\mathbf{1 0 5}$ healthy subjects

Presenting Author: Vilma Beleskiene

Vilma Beleskiene

Vilnius University Hospital Santariskiu Clinics

\section{Learning Objectives:}

Objectives: The aim of this study was to establish the rate variation of sonotubometric measurements using a specific broadband class of signals, the so-called perfect sequences (PSEQ) among healthy adults and to identify an optimal and technically simple test to provoke Eustachian tube (ET) openings.

Methods: Sonotubometry was performed on 105 healthy adult subjects. Three different consecutive maneuvers were performed for ET opening: dry swallowing, water swallowing (a small $(2 \mathrm{ml})$ and a large $(5 \mathrm{ml})$ water bolus). Values of the amplitude and duration of each measured ET opening were calculated.

Results: 6,300 measurements were performed. Sonotubometric ET openings were detected for all subjects but not for each measurement. 6,180/6,300 measurements (98.1\%) objective ET openings were registered. Mean ET opening duration time and the mean sound amplitude were similar for all performed test and are $270(\mathrm{SD} \pm 96) \mathrm{ms}$, $13.48(\mathrm{SD} \pm 6.57) \mathrm{dB}$.

Conclusion: sonotubometry based on PSEQ stimuli is a reliable methodology to assess the Eustachian Tube opening function in healthy subjects. Mean ET opening duration time and the mean sound wave amplitude were similar performing all analysed tests, hence might be concluded, that dry (saliva) and water swallowing are a reliable sonotubometric maneuvers and may be used examining ET opening function. Size of a sip during water swallowing does not affect the sonotubometry result. All maneuvers can be equally used as the optimal maneuver, and we think that water swallow is most comfortable for the subject.

\section{doi:10.1017/S0022215116005107}

\section{ID: IP014}

Devices for restoring hearing in the sequelae of cholesteatoma
Presenting Author: Millo Achille Beltrame

Millo Achille Beltrame

Centro Clinico Multispecialistico

Rovereto

Learning Objectives: Possible solution to restore the hearing using bone conduction implants and middle ear implants solutions. The presentation illustrates different situations, in patients where the hearing loss changed post cholesteatoma's surgery.

The hearing loss in patients with history of Cholesteatoma,have multifarious features that depend either by the damaging disease activity or by the result of the surgery. The majority of them suffer from conductive hearing losses because the ossicles have been destroyed by the disease or surgically removed. The hearing has to be restored, reconstructing the ossicular chai$\mathrm{n}$ (ossiculoplasty)or, in other cases, with hearingimplants. Frequently patients with previous cholesteatoma, show a mixed hearing loss, due to the aging or toxicity of the disease. A limited number presents a profound deafness, or anacusis due to a cholesteatoma's invasion into the labyrinth or by iatrogenic damage during the surgery. All these outcomes can be corrected with auditory implants inserted either in the middle ear or in the cochlea. Subjects that have CWU and CWD tympanoplasty done and good bone hearing threshold were treated with BONEBRIDGE device in the retro-sigmoid site, in order to avoid any future contamination in case of a disease recurrence or infections in the middle ear. In mixed hearing losses, a Vibrabt Soundbridge system has been preferred to place the FMT over the stapes, if present, or onto the footplate or on the round window. In CWD cases the round window membrane has been always the site of choice. When the cavity is completely clean and dry a VSB could be implanted with the FMT on the $\mathrm{RW}$ pays attention of the positioning of the conductor link, far from the diseased area and in a channel covered with bone pâté. Doing a cholesteatoma removal and a middle ear implantation on the same surgical session is not always the best option. There are situations where the procedure requires two steps: cholesteatoma removal first and implantation later. Always an open external ear canal is mandatory in order to check with otoscopy the condition of the middle ear cavity in the next visits.Finally,in patients with profound hearing losses, a cochlear implant was implanted via retro-sub-facial approach.

doi:10.1017/S0022215116005119

\section{ID: IP015}

The Chorda Tympani Nerve Degenerates during Chronic Otitis Media. An Electrone Microscopy Study

\section{Presenting Author: Katarina Berling Holm}

Katarina Berling Holm ${ }^{1}$, Magnus von Unge ${ }^{2}$, Paula Mannström ${ }^{3}$, Mats Ulfendahl ${ }^{3}$, Niklas Danckwardt Lillieström ${ }^{4}$

${ }^{1}$ Center for Clinical Research, Landstinget Västmanland, Västerås, Sweden, ${ }^{2}$ Dept. of Otorhinolaryngology, Akershus University Hospital and University of Oslo, Norway, ${ }^{3}$ Dept. of Neuroscience, Karolinska Institutet, 